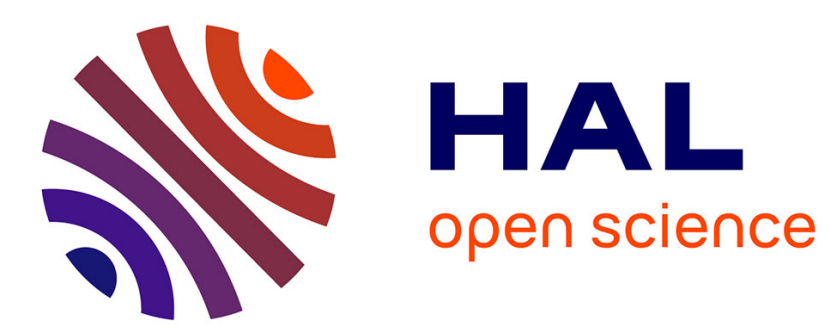

\title{
Attack-tolerant Unequal Probability Sampling Methods over Sliding Window for Distributed Streams
}

\author{
Yann Busnel, Yves Tillé
}

\section{To cite this version:}

Yann Busnel, Yves Tillé. Attack-tolerant Unequal Probability Sampling Methods over Sliding Window for Distributed Streams. ICCDA 2020 : 4th International Conference on Compute and Data Analysis, Mar 2020, San Jose, United States. pp.72-78, 10.1145/3388142.3388162 hal-02456880

\section{HAL Id: hal-02456880 https://imt-atlantique.hal.science/hal-02456880}

Submitted on 28 Jan 2020

HAL is a multi-disciplinary open access archive for the deposit and dissemination of scientific research documents, whether they are published or not. The documents may come from teaching and research institutions in France or abroad, or from public or private research centers.
L'archive ouverte pluridisciplinaire HAL, est destinée au dépôt et à la diffusion de documents scientifiques de niveau recherche, publiés ou non, émanant des établissements d'enseignement et de recherche français ou étrangers, des laboratoires publics ou privés. 


\section{Attack-tolerant Unequal Probability Sampling Methods over Sliding Window for Distributed Streams}

\author{
Yann Busnel \\ IMT Atlantique \\ IRISA, UMR CNRS 6074 \\ Rennes, France \\ Yann.Busnel@imt-atlantique.fr
}

\author{
Yves Tillé \\ Université de Neuchâtel \\ Institut de statistique \\ Neuchâtel, Switzerland \\ Yves.Tille@unine.ch
}

January 28, 2020

\begin{abstract}
Distributed systems increasingly require the processing of large amounts of data, for metrology, safety or security purposes. The online processing of these large data streams requires the development of algorithms to efficiently calculate parameters. If elegant solutions have been proposed recently, their approximation is commonly calculated from the inception of the data stream. In a distributed execution context, it would be preferable to collect information only on the recent past (for resource saving or relevancy of most recent information). We therefore consider here the sliding window model.
\end{abstract}

In this article, we propose a family of new sampling techniques that take into account both the sliding window model and the presence of a malicious adversary. Wayne Fuller proposed in 1970 a very ingenious method of sampling with unequal inclusion probabilities. After doing justice to this precursor paper and proposing a fast and simple implementation of it, we completely generalize Fuller's method in order to enable the use of a tuning parameter of spreading. The analytical results of these techniques show the excellent performance of the generalized pivotal approach. This generalization makes the sampling method less predictable and seems appropriate to be protected from malicious attacks when sampling from a stream.

Keywords: Sampling algorithm, Sliding window, Byzantine adversarial, Brewer method, Pivotal method.

\section{Introduction and related works}

In very large distributed network management systems, it is often critical to collect global data given the very large number of participants. This can be modeled by a set of nodes, each observing a data stream. These nodes must therefore collaborate to continuously evaluate a given function on the global distributed stream. For example, current network management tools analyze the input streams of a set of routers to detect malicious sources or to extract user behavior [2, 21]. The main objective is to evaluate these functions at the lowest cost in terms of space used on each node, as well as update and query time. The prob- lem of extracting relevant information from a (distributed) data stream(s) is similar to the problem of identifying patterns that do not conform to the expected behaviour. This theme has been a very productive field of research in recent decades. For example, depending on the specificities of the domain and the type of aberrations considered, different methods have been designed: classification, grouping, nearest neighbour, spectral statistics and information theory. An interesting state of the art on these techniques, describing their advantages and disadvantages, is proposed by [10. A common feature of these techniques is their high complexity in terms of space and computation cost, as they are based on algorithms with open memory, which require complete knowledge and access to the analyzed data.

Relying on algorithms of this type is not feasible in a context of real-time information extraction, with very low storage and processing capacity. However, two main approaches exist for real-time processing of massive, potentially distributed data streams.

The first approach is to process all the data in the stream on the fly, and to keep only summaries, or aggregates, locally, in which only essential information about the data is kept [10]. This approach extracts statistics on processed data streams, with bounded error probabilities, without assuming any constraint on the order in which the data is received on the nodes (i.e., the data order could be manipulated by an omnipotent opponent [4]). Most existing research using this approach has focused on calculating statistical functions or measurements with a given $\varepsilon$ error, using a poly-logarithmic memory amount in the size of the stream and domain of the incoming data. These functions estimate, for example, the number of distinct data present in a given stream [23, frequency moments [1, the most frequent elements [11, 28, entropy of a stream [9], flood attack detection [30, the correlation between several streams [3] or the relative entropy between a biased stream and a uniform stream [2, 5. Unfortunately, these estimates are based on summaries that involve a significant loss of information, with sometimes drastic overestimation (as in the case of the Count-Min Sketch [14).

On the other hand, the second consists in regularly sampling the stream, making it possible to limit the amount of data stored in memory [1, 24, 25, 26]. This method allows 
to calculate exactly functions on these samples. However, the reliability of this calculation, in comparison with the exact result calculated on the entire stream, depends strongly on the volume of data that has been sampled, and the position of this data on the stream. Worse still, an opponent could easily take advantage of the sampling policy to hide his attacks among the unsampled packets, or thereby prevent his "malicious" packets from being correlated. The primary objective of this article is to further take these two characteristics into account by using methods from survey theory.

In addition, the majority of current solutions offer sampling methods over the entire stream. The use of these techniques, for example in network monitoring, requires the selection of samples from recent data only. Carrying the well-known sampling solutions in a sliding window is often complex or even impossible given the very nature of the sampling process. In addition, classical methods do not necessarily take into account the dispersion of the sampled elements in the current window. Indeed, without stratification, the samples can be spread too evenly, or conversely concentrated in certain parts of the input stream. Adversaries could take advantage of this knowledge to place their samples in such a way as to bias the overall sampling. The emergence of the pivotal method in survey theory suggests a relevant solution to these problems.

In a manuscript paper written in French, Deville [17. proposed an unequal probability sampling method that we refer to as the "Deville systematic method". The method is also described in detail in Tillé [31, pp. 128-130]. Another unequal probability sampling procedure is the pivotal method proposed by Deville and Tillé [18] in the framework of the splitting method [see also 31, pp. 106-08]. When the pivotal method is applied according to the order of the units, it is usually called "sequential pivotal method" or "ordered pivotal method". In a relatively technical paper, Chauvet 12. showed that the ordered pivotal method and Deville's systematic method are two algorithms that implement the same sampling design, which is certainly not obvious when comparing the two methods.

Fuller [20] proposed a method where one unit is sampled in each stratum but with random strata boundaries. The method is developed for equal and unequal probability sampling. Fuller's method is no other than Deville's Systematic method with random strata boundaries.

\section{Contributions and plan of the pa- per}

Our main contributions are the following. We first propose a very simple implementation of Fuller's method by means of the pivotal method. Next we generalize the pivotal and the Fuller methods. A tuning parameter is introduced that enables to choose the spreading of the sample. A large family of methods is then defined for the cases where the variable of interest is autocorrelated. In brief, these methods make it possible to achieve the objectives of well-spread sampling, with computational complexity and minimal memory cost, while respecting the constraints of the aforementioned model.

In addition, to be able to theoretically study the impact of opponents on the algorithms proposed in the literature, it is possible to use different models from the distributed systems community. For example, as Anceaume et al. in [7], we consider the presence of an opponent adaptive, who tries to avoid the smooth running of the algorithm by distributing his data judiciously among the different streams. It is thus able to insert as much information as necessary to increase or reduce the accuracy of an algorithm, in relation to the current state of the system, or to reorder the streams as it sees fit. However, generally, it is considered that any algorithm run by any correct node is public knowledge to avoid some kind of security by obscurity.

\section{System model}

We consider a set $\mathcal{S}$ of $\ell$ nodes $S_{1}, \ldots, S_{\ell}$ such that each node $S_{i}$ receives a large sequence $\sigma_{S_{i}}$ of data items or units. We assume that streams $\sigma_{S_{1}}, \ldots, \sigma_{S_{\ell}}$ do not necessarily have the same size, i.e., some of the items present in one stream do not necessarily appear in others or their occurrence number may differ from one stream to another one. We also suppose that node $S_{i}(1 \leq i \leq \ell)$ does not know the length of its input stream. Items arrive regularly and quickly, and due to memory constraints (i.e., nodes can locally store only a small amount of information with respect to the size of their input stream and perform simple operations on them), need to be processed sequentially and in an online manner. Nodes cannot communicate among each other. On the other hand, there exists a specific node, called the coordinator in the following, with which each node may communicate [15. We assume that communication is instantaneous. We refer the reader to [29] for a detailed description of data streaming models and algorithms. Note that in the some contexts such as IoT or edge computing, it may not be reasonable to rely on a central entity. We could extend our distributed solution to a fully decentralized version by organizing sites in such a way that each one could locally aggregate the information provided by its neighbours, as done in [6].

\section{The pivotal method}

Let's consider a $U$ population of size $N$, representing all the information that can be exchanged within the $\mathcal{S}$ network, in which we want to select a sample of fixed size $n$ with unequal inclusion probabilities $0<\pi_{k}<1, k \in U$, such that $\sum_{k \in U} \pi_{k}=n$. The pivotal method is particularly simple and consists of picking at each step two units (denoted by $i$ and $j$ ) in the population. Their inclusion probabilities $\left(\pi_{i}, \pi_{j}\right)$ are randomly transformed to $\left(\tilde{\pi}_{i}, \tilde{\pi}_{j}\right)$ using the following randomization:

$\left(\tilde{\pi}_{i}, \tilde{\pi}_{j}\right)=\left\{\begin{array}{r}\left(\min \left(1, \pi_{i}+\pi_{j}\right), \max \left(\pi_{i}+\pi_{j}-1,0\right)\right) \\ \text { with probability } q \\ \left(\min \left(\pi_{i}+\pi_{j}, 1\right), \max \left(0, \pi_{i}+\pi_{j}-1\right)\right) \\ \text { with probability } 1-q\end{array}\right.$ 
with

$$
q=\frac{\min \left(1, \pi_{i}+\pi_{j}\right)-\pi_{j}}{2 \min \left(1, \pi_{i}+\pi_{j}\right)-\pi_{i}-\pi_{j}} .
$$

The validity of the method is based upon the fact that the expectation of this pair corresponds to the inclusion probabilities, i.e., $\mathrm{E}\left(\widetilde{\pi}_{i}, \widetilde{\pi}_{j}\right)=\left(\pi_{i}, \pi_{j}\right)$ and $\widetilde{\pi}_{i}+\widetilde{\pi}_{j}=\pi_{i}+\pi_{j}$. This ensures that the inclusion probabilities are satisfied and that the sum of the components of the vector is always equal to $n$.

This elementary step can be repeated on couples of units containing values that are not equal to 0 or 1 . Since at each step, a component is set to 0 or 1 , in $N-1$ steps at most, the sample is selected. The ordered pivotal method simply consists in taking the units according to their order in the vector of inclusion probabilities, which is built on the fly according to the incoming stream. So the first step is applied on units $(1,2)$. The unit that conserves a noninteger value is then confronted to unit 3 and so on.

If the sum of the inclusion probabilities is not an integer, the pivotal method ends up with a vector whose components are all integer except for one that is equal to the fractional part of the sum of the inclusion probabilities. This result is given for instance by the UPpivotal function of the $\mathrm{R}$ sampling package, which was proposed by the authors 27. The pivotal method is a particular case of the cube method [19] with only one balancing variable that is equal to the inclusion probabilities. The ordered pivotal method is the particular case of the fast implementation of the cube method proposed by Chauvet and Tillé [13].

\section{Implementation of Fuller's method}

In Fuller's method as in Deville's systematic method, the main ideas consist of constructing strata and selecting one unit in each stratum. The main problem is that it is almost always impossible to construct strata in which the sums of the inclusion probabilities are equal to 1 . In Deville's method, one first computes the cumulated inclusion probabilities

$$
V_{k}=\sum_{i=1}^{k} \pi_{k} \text { with } V_{0}=0, \text { and } V_{N}=n .
$$

The strata are the intervals $[0,1],[1,2], \ldots[n-1, n]$. A unit $k$, such that the interval $\left[V_{k-1}, V_{k}\right]$ contains an integer, is called a boundary unit, because it belongs to two consecutive strata. The main difficulty of Deville's systematic method consists of avoiding selecting twice the boundary units. Drawing probabilities must be recomputed in each stratum in function of the selection in the previous stratum. The algorithm and the procedure of updating the drawing probabilities are described in detail in Tillé [31, p.130].

Fuller's method is exactly the same as Deville's systematic sampling except for the computation of the bounds of the strata. In Deville systematic sampling, the bounds are the integer numbers $0,1,2, \ldots, n$. In Fuller's method,

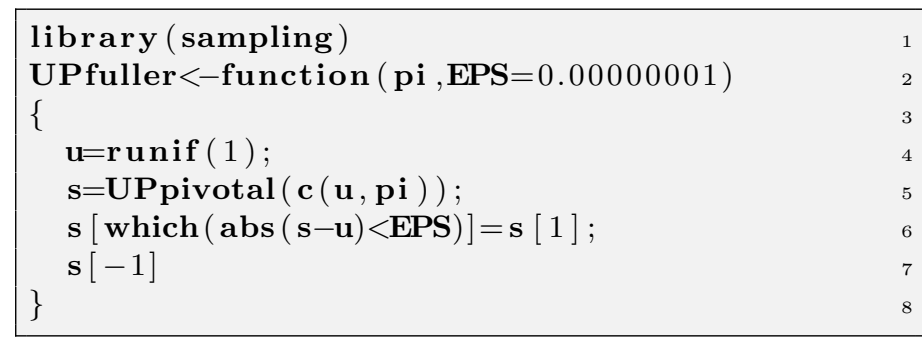

Listing 1: Implementation of Fuller's method

the first bound is randomly selected using a uniform random variable $u$ in $[0,1]$. The bounds of the strata are thus $0, u, u+1, u+2, \ldots, n-1+u, n$. Fuller's method has one more stratum, but the algorithm begins by deciding randomly if a unit is selected in stratum $[0, u]$ or in $[n-1+u, n]$, which is equivalent to considering that these two intervals form a single stratum.

Chauvet [12] showed that Deville's systematic method and the ordered pivotal method exactly implement the sample sampling design. Fuller's method can also be implemented by the pivotal method provided that a random start is used ( $c f$. Listing 1). Suppose that we have a vector of inclusion probabilities which sum up to an integer. First we randomly create a phantom unit whose inclusion probability $\pi_{0}$ is a uniform random variable in [0,1] (line 3). Next the ordered pivotal method is applied on this population of size $N+1$ (line 4). Since the sum of the inclusion probabilities is equal to $n+\pi_{0}$, the random pivotal method returns a vector whose components are all equal to 0 or 1 except for one (denoted by $j$ ) that is equal to $\pi_{0}$. Finally, unit $j$ is selected if the phantom unit has been selected and is not selected otherwise. The phantom unit is then removed from the sample. If the phantom unit remains with the non-integer value, it is simply removed from the sample. The code of Listing 1 gives the $\mathrm{R}$ implementation that is particularly simple.

\section{Generalization of the Pivotal and Fuller's method}

\subsection{Main idea}

The pivotal method and the Fuller method can be generalized. In place of confronting two units, one can consider three or more units. Suppose that $r$ consecutive units are considered at each step. These units can be randomly transformed in such a way that one of them is transformed into a 0 or a 1 . This transformation can be done by several procedure. We propose three possible methods to transform the subsets.

\subsection{Brewer's method}

Brewer 8 proposed a very interesting unequal probability method. Tillé [31, pp. 112-114] reformulate the method as a particular case of the splitting method. For the generalization of the pivotal method, the method must be extended 


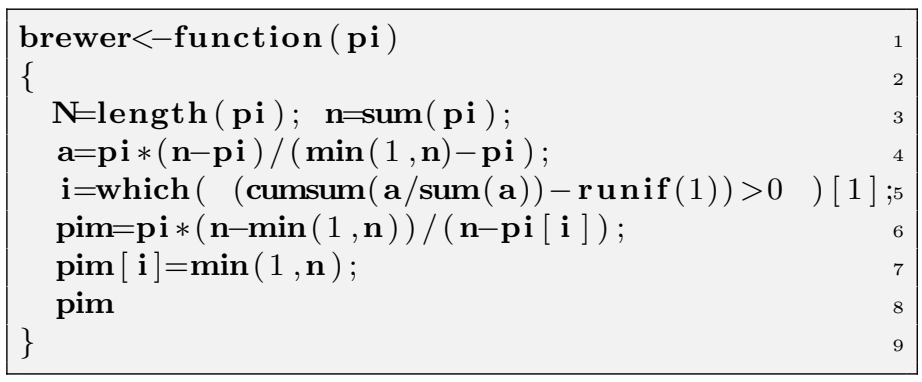

Listing 2: Implementation of Brewer's method

to the case where the sum of the inclusion probabilities is non-integer.

The basic step of the Brewer method can be expressed as follows:

- Select an integer number (say $\ell$ ) between 1 and $N$ with probabilities ( $c f$. lines 4 and 5 of Listing 2):

$$
\alpha_{j}=\left(\sum_{i \in U} \frac{\pi_{i}\left(n-\pi_{i}\right)}{\min (1, n)-\pi_{i}}\right)^{-1} \frac{\pi_{j}\left(n-\pi_{j}\right)}{\min (1, n)-\pi_{j}}, j \in U .
$$

- Change the vector of inclusion probabilities, for all $k \in$ $U$, into ( $c f$. lines 6 and 7 of Listing 2):

$$
\pi_{k}^{\ell}= \begin{cases}\frac{\pi_{k}(n-\min (n, 1))}{n-\pi_{\ell}} & \text { if } k \neq \ell \\ \min (n, 1) & \text { if } k=\ell .\end{cases}
$$

The method is valid because $0 \leq \pi_{k}^{\ell} \leq 1$ for all $\ell \in U, k \in$ $U$,

$$
\sum_{k \in U} \pi_{k}^{\ell}=n, \ell \in U, \text { and } \sum_{\ell \in U} \alpha_{\ell} \pi_{k}^{\ell}=\pi_{k}, k \in U .
$$

The short $\mathrm{R}$ code of Listing 2 gives an implementation.

Brewer's method has however a drawback when it is used to generalize the pivotal method. When $n=\sum_{k \in U} \pi_{k}<1$, then all the $\pi_{k}^{\ell}$ are null except one that is equal to $n$. There are then null joint inclusion probabilities. For this reason we propose to use the complementary Brewer method.

\subsection{Complementary Brewer method}

A complementary sampling design $p^{c}(s)$ of a sampling design $p(s)$ is defined as follows:

$$
p^{c}(s)=p(U \backslash s), s \subset U .
$$

The complementary of the Brewer method can be derived directly from the previous section. The basic step of the complementary Brewer method will thus transform one component in a zero at each step.

- Select an integer number (say $\ell$ ) between 1 and $N$ with probabilities ( $c f$. lines 4 and 5 of Listing 3 ):

$$
\begin{aligned}
\alpha_{j}= & \left(\sum_{i \in U} \frac{\left(1-\pi_{i}\right)\left(N-n-1+\pi_{i}\right)}{\min (1, N-n)-1+\pi_{i}}\right)^{-1} \\
& \times \frac{\left(1-\pi_{j}\right)\left(N-n-1+\pi_{j}\right)}{\min (1, N-n)-1+\pi_{j}}, j \in U .
\end{aligned}
$$

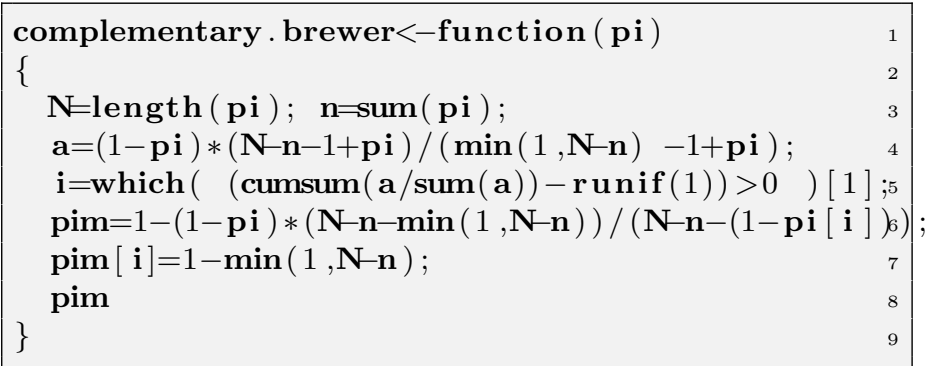

Listing 3: Implementation of Complementary Brewer's method

- Change the vector of inclusion probabilities into ( $c f$. lines 6 and 7 of Listing 2):

$\pi_{k}^{\ell}= \begin{cases}1-\frac{\left(1-\pi_{k}\right)(N-n-\min (N-n, 1))}{N-n-1+\pi_{\ell}} & \text { if } k \neq \ell \\ 1-\min (1, N-n) & \text { if } k=\ell,\end{cases}$

for all $k \in U$.

Again, the method is valid because

$$
0 \leq \pi_{k}^{\ell} \leq 1 \text { for all } \ell \in U, k \in U
$$

$$
\sum_{k \in U} \pi_{k}^{\ell}=n, \ell \in U, \text { and } \sum_{\ell \in U} \alpha_{\ell} \pi_{k}^{\ell}=\pi_{k}, k \in U .
$$

The short $\mathrm{R}$ code of Listing 3 gives an implementation.

\subsection{Random direction method}

Another method consists of generating a random vector of size $N$ with null mean. The code of Listing 4 gives an implementation of this method.

For instance, $N$ standardized random variables $v_{1}, \ldots, v_{n}$ are generated and next are centered (line 4):

$$
u_{k}=v_{i}-\frac{1}{N} \sum_{i \in U} v_{i}
$$

Next, one searches the maximum value $\lambda_{1}$ and the minimum value $\lambda_{2}$ that satisfy

$$
0 \leq \pi_{k}+\lambda_{1} u_{k} \leq 1 \text { and } 0 \leq \pi_{k}+\lambda_{2} u_{k} \leq 1 \text { for all } k \in U .
$$

This is computed between lines 5 and 7 .

Finally, the vector of $\pi_{k}$ is replaced by

$$
\pi_{k}^{n e w}=\left\{\begin{array}{l}
\pi_{k}+\lambda_{1} u_{k} \text { with probability }-\frac{\lambda_{2}}{\lambda_{1}-\lambda_{2}} \\
\pi_{k}+\lambda_{2} u_{k} \text { with probability } \frac{\lambda_{1}}{\lambda_{1}-\lambda_{2}}
\end{array}\right.
$$

(cf. lines 8-11).

As in sections above, the method is also valid because $\mathrm{E}\left(\pi_{k}^{n e w}\right)=\pi_{k}$ and $0 \leq \pi_{k}^{n e w} \leq 1$ for all $k \in U$.

\subsection{General algorithm: multivotal method and generalized Fuller method}

The three methods described in the previous sections transform randomly one of the components of the vector into a 


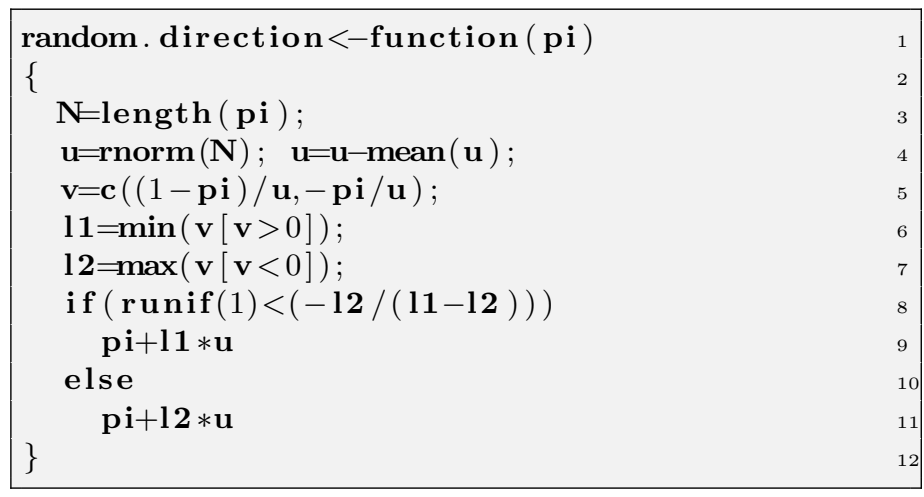

Listing 4: Implementation of the Random direction method

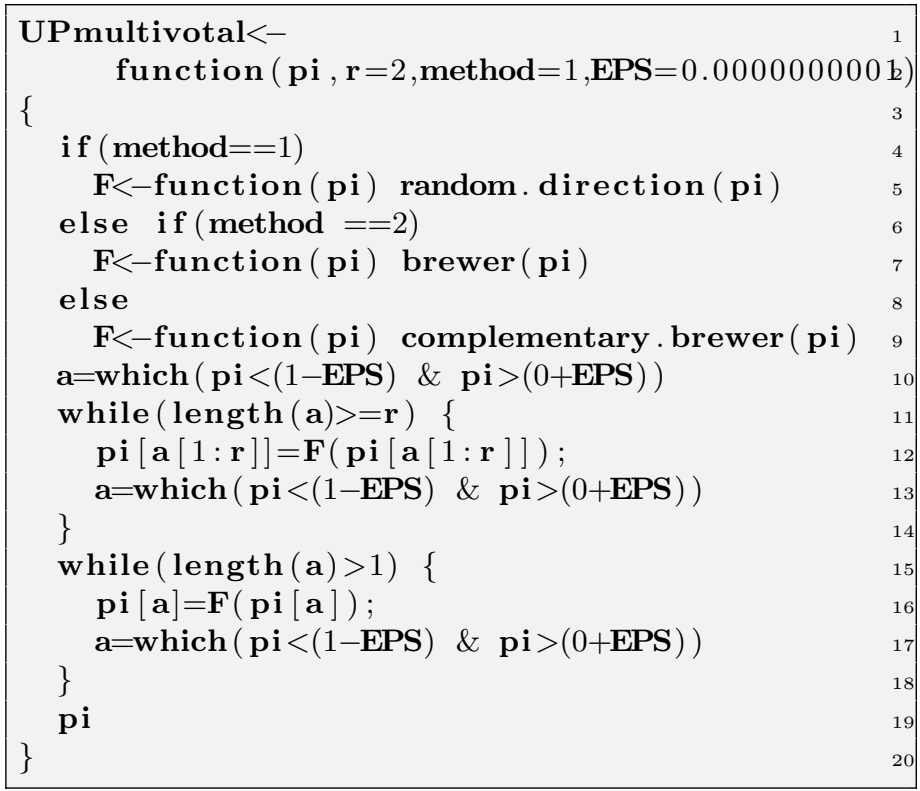

Listing 5: Implementation of the Multivotal method

0 or a 1 . When these methods are applied on a vector of size 2, they are all equal to the pivotal method.

Now, the pivotal method can be generalized. At the first step, the first $r \geq 2$ units are selected. On this subset of $r$ units, one of the three methods (Brewer, Complementary Brewer or Random direction) is applied on this subset. One of the components of this subset is transformed into a 0 or a 1 . Next, the units that are not integer of this subset are completed by the following units of the stream in order to constitute again a subset of $r$ units. Again, one of the three methods is applied and so on.

Listing 5 propose an implementation of this method. Lines 4 to 9 choose the method applied. Line 10 select non integer units from the stream, then Lines 11 to 14 apply the aforementioned method to transform one unit either in 0 or 1 , one after the other. Finally, when less than $r$ units remain non integer, the chosen method finalize the tranformation (lines 15-18).

Finally Fuller's method can be generalized by using the same trick of the phantom unit used for the pivotal method. Listing 6 proposes an implementation in R language. Line 4 uses the aforementioned trick, as line 6 applies the Multiv-

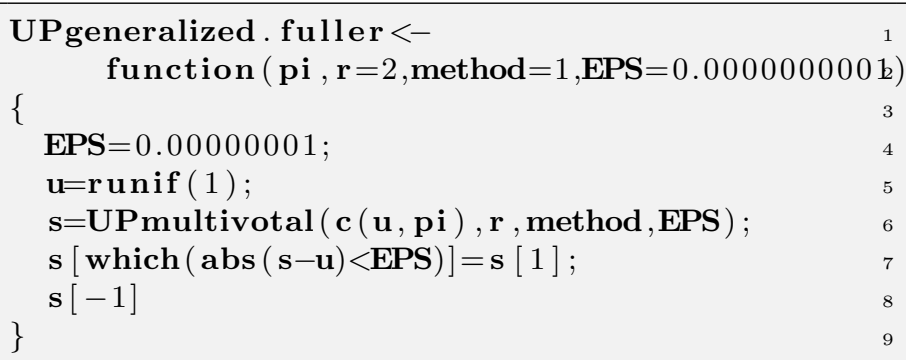

Listing 6: Implementation of the Generalized Fuller's method

otal method on this modified vector.

\section{$7 \quad$ Simulations}

In order to simulate the behavior of these methods on data streams, an artificial population of size $N=200$ has been generated with heteroscedasticity and autocorrelation. First an auto-correlated variable has been created by $\varepsilon_{k}=0.9 \varepsilon_{k}+u_{k}$ where $\varepsilon_{1}$ and $u_{k}(k=2, \ldots, N)$ are independent standardized normal random variables. Next the $x_{k}$ are independent Gamma variables with shape 5 , rate $=0.25$. The interest variables are $y_{k}=x_{k}\left(4+\varepsilon_{k}\right)$. The sample size is set to $n=50$ and the inclusion probabilities are proportional to $x_{k}$.

A set of 200,000 simulations is run to approximate the variances of the different methods. Then, to evaluate the quality of each sample provided by these methods, the Hájek estimators are next computed 22]:

$$
\widehat{Y}=\frac{\sum_{k \in U} \frac{y_{k} a_{k}}{\pi_{k}}}{\sum_{k \in U} \frac{a_{k}}{\pi_{k}}},
$$

where $a_{k}=1$ if $k$ is selected in the sample and 0 otherwise.

We consider the variance of the Hájek estimator for the random pivotal method as the baseline of our comparisons. This variance is equal to $39.14376(100 \%)$. The variance of the pivotal method equals $25.09(64.07 \%)$, that is $64.07 \%$ of the variance of the random pivotal method. More interestingly, for the Fuller method, the variance equals 26.67 $(68.13 \%)$.

Table 1 contains the ratios of variances of $\widehat{Y}$ for the other method and different values of $r$ with respect to the random pivotal method. When $r=2$, all the methods reduce to the pivotal or the Fuller method. Unsurprisingly, when $r$ increases, the variance increases. Figure 1 illustrates the results presented in Table 1. Moreover the "Brewer" methods are a little more accurate. This is probably due to the fact that the probabilities are more likely to be set to zero during the algorithm. The Brewer methods are thus more repulsive.

Figures 2 and 3 contain examples (with $N=20, n=7$ and equal inclusion probabilities) where $\pi_{k \ell} /\left(\pi_{k} \pi_{\ell}\right)$ is plotted in function of $|k-\ell|$ for different values of $r$. This corresponds to the normalized second order probabilities (divided by the first order probabilities). The larger $r$, the more $\pi_{k \ell} /\left(\pi_{k} \pi_{\ell}\right)$ are equal. Thus, if $r$ increases, the joint 
Table 1: Variances of the Hájek estimators for the different sampling designs over 200,000 simulations.

Results are given for the multivotal method (Mult.) and Generalized (G.) Fuller method with the three possibilities: Random direction, Brewer, and Complementary (Comp.) Brewer method.

\begin{tabular}{ccccccc}
\hline & \multicolumn{2}{c}{ Random direction } & \multicolumn{2}{c}{ Brewer } & \multicolumn{2}{c}{ Compl. Brewer } \\
\hline$r$ & Mult. & G. Fuller & Mult. & G. Fuller & Mult. & G. Fuller \\
\hline \hline 2 & 63.65 & 68.13 & 63.89 & 67.89 & 63.88 & 68.11 \\
3 & 70.82 & 71.42 & 67.37 & 68.53 & 67.15 & 70.96 \\
4 & 74.47 & 74.70 & 69.94 & 69.97 & 73.22 & 73.72 \\
5 & 77.30 & 77.20 & 70.32 & 68.76 & 75.95 & 77.38 \\
6 & 79.51 & 80.12 & 73.73 & 73.79 & 77.21 & 78.32 \\
7 & 82.79 & 82.10 & 72.03 & 71.99 & 82.09 & 80.75 \\
8 & 84.01 & 83.35 & 76.63 & 75.26 & 82.56 & 81.87 \\
9 & 85.35 & 85.66 & 77.96 & 75.66 & 84.89 & 84.11 \\
10 & 86.56 & 86.59 & 80.42 & 79.79 & 86.71 & 85.30 \\
11 & 88.27 & 88.11 & 80.36 & 80.10 & 84.83 & 83.77 \\
12 & 89.44 & 88.98 & 76.01 & 75.06 & 88.98 & 87.76 \\
\hline
\end{tabular}

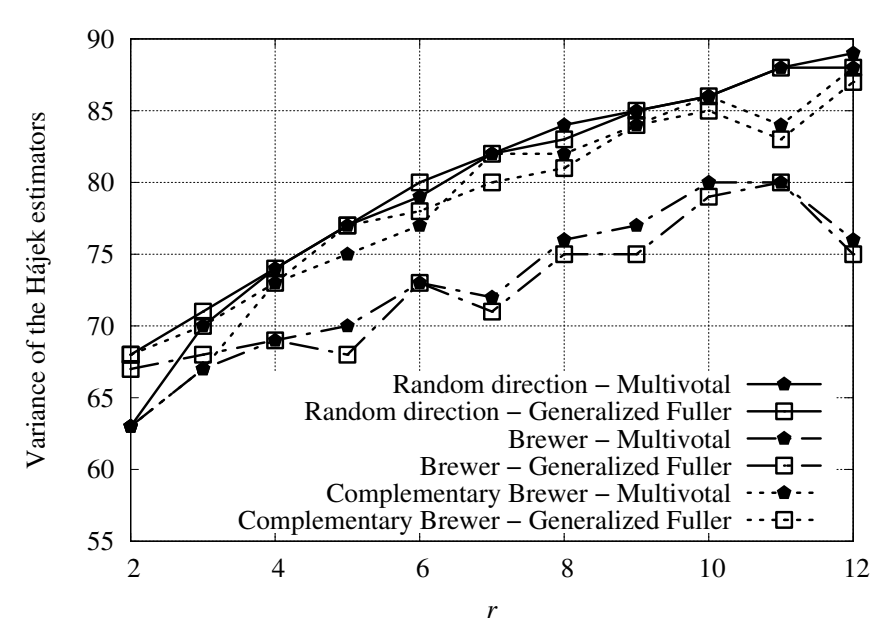

Figure 1: Variances of the Hájek estimators for the different sampling designs over 200,000 simulations.

Results are given for the multivotal method (Mult.) and Generalized (G.) Fuller method with the three possibilities: Random direction, Brewer, and Complementary (Comp.) Brewer method.

inclusion probabilities tends to a situation of independence, which is the less predictable situation. In other words, if these probabilities are constant, it means that selecting one unit does not provide information on selecting the next. This property is particularly interesting in the presence of a powerful adversary. Indeed, the latter will not be able to take advantage of the knowledge of the last sampled items to place his own malicious items knowingly.

On both figures, one can observe that when $r=2$, selecting the unit makes it very unlikely to select the next unit. When $r$ is higher, the probabilities tend to equalize. This makes it much harder to predict what will happen next.

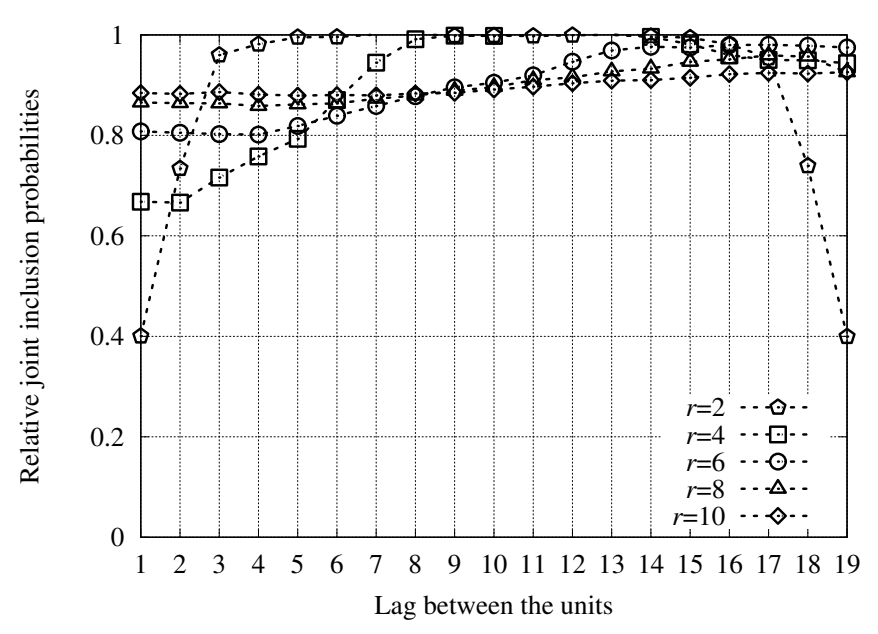

Figure 2: Relative joint inclusion probabilities, over 1,000,000 simulations, for the Generalized Fuller method with the Complementary Brewer method

\section{Discussion and extensions}

Wayne Fuller has been really a precursor in the sense that he proposed a method that is more general by using random boundaries. His paper is very technical and the implementation can be simplified. Fuller's method is however an excellent procedure. The sample is spread by the use of strata with random boundaries. The new implementation enables to define a fast and sequential algorithm. The joint inclusion probabilities are not null, which is an advantage with respect to the use of nonrandom boundaries. A simple expression for the joint inclusion probabilities remains to be found and seems to be really challenging. The pivotal method can also be generalized by confronting more than two units at each step. This generalization makes the sam- 


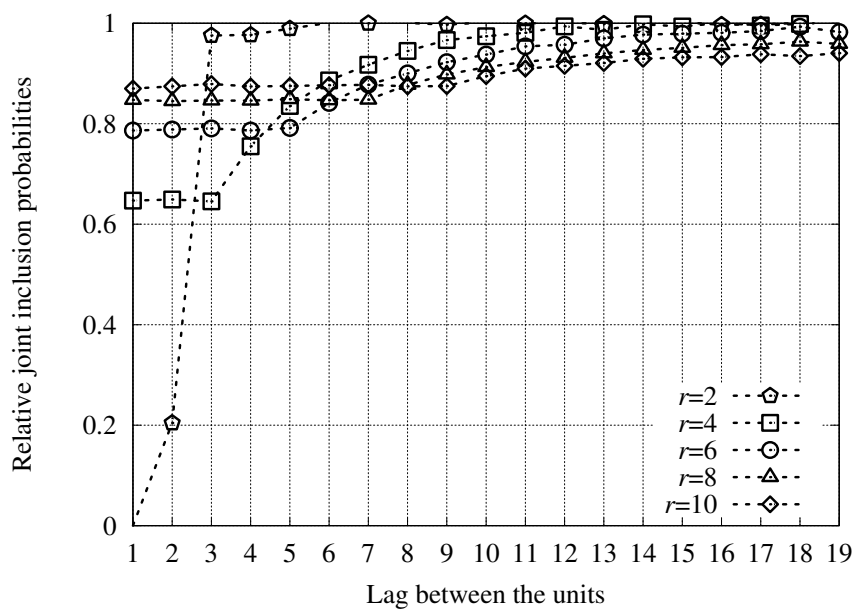

Figure 3: Relative joint inclusion probabilities, over 1,000,000 simulations, for the Multivotal method with the Random direction method

pling method less predictable and could be appropriate to be protected from malicious attacks when sampling from a stream.

In one hand, if $r$ equals 2 all the methods reduce to the pivotal method. On the other hand, if $r$ equals $N$, the procedure reduces the method of Brewer, complementary Brewer or random direction. In this last case all the units are involved in the next step of the procedure. When $r=2$, the next step is very predictable because the next decision will only concern two units. If $r=N$, nothing can predict which units will be selected from the following $N$. Parameter $r$ can be used to tune the design. The larger $r$, the most unpredictable is the selection of the following units. However the smaller $r$, the more accurate is the estimator. Between these two values a tradeoff can be find according to the considered problem.

In addition, all these algorithms allow to take into account the needs of sliding windows. The sliding window model formalized by Datar et al. [16] defines the validity period of an element, i.e., the sample will only be drawn from the more recent $N^{\prime}<<N$ elements among the $U$ elements already observed. In this model, the samples arrive continuously and expire after exactly the $N^{\prime}$ steps. A step corresponds to the arrival of an element of the population, we consider sliding windows based on counting. The challenge is to perform this calculation in the sublinear space. When $N^{\prime}$ is set to the maximum value $N$, the sliding window model is reduced to the classic model. The additional problem with a sliding window is that when a prefix of a stream is summarized, we lose the time information related to the different elements, making the exclusion of the oldest elements non-trivial with little memory. The interest of the methods presented above relies in the inherent stratification of sampling. Also, it is sufficient to keep the samples taken in the last $N^{\prime}$ strata to make the method reliable, efficient and robust in a sliding window.

Finally, we propose an algorithmic adaptation that allows sampling according to any of the techniques presented above on a set of $\ell$ distributed data streams, so that the number of bits communicated between the $\ell$ sites and the coordinator is minimal. This amounts to the coordinator gathering all the samples on the previous window. The union then represents a meaningful sample of the overall distributed stream. As mentioned earlier in Section 3 it is possible to have a fully decentralized version of our algorithm, for example by organizing nodes within a distributed hash table (DHT) and by taking advantage of the additive property of the samples to allow each site to collect its pattern and gradually obtain a global view of the current sample. Such a possible solution appears in 6]. It should be noted, however, that the distributed version would have a significant impact on the cost of communication. This issue is left open for future work.

\section{References}

[1] N. Alon, Y. Matias, and M. Szegedy. 1996. The space complexity of approximating the frequency moments. In Proceedings of the 28th ACM Symposium on Theory of computing (STOC). 20-29.

[2] E. Anceaume and Y. Busnel. 2014. A Distributed Information Divergence Estimation over Data Streams. IEEE Transaction on Parallel and Distributed System 25, 2 (2014), 478-487. https://doi.org/10.1109/ TPDS.2013.101

[3] E. Anceaume and Y. Busnel. 2017. Lightweight Metric Computation for Distributed Massive Data Streams. Transaction on Large-Scale Data- and KnowledgeCentered Systems 33 (2017), 1-39.

[4] E. Anceaume, Y. Busnel, and S. Gambs. 2010. Uniform and Ergodic Sampling in Unstructured Peer-toPeer Systems with Malicious Nodes. In Proceedings of the 14th International Conference on Principles of Distributed Systems (OPODIS), Vol. 6490. 64-78. https://doi.org/10.1007/978-3-642-17653-1_5

[5] E. Anceaume, Y. Busnel, and S. Gambs. 2012. AnKLe: Detecting Attacks in Large Scale Systems via Information Divergence. In Proceedings of the 9th European Dependable Computing Conference (EDCC). https://doi.org/10.1109/EDCC.2012.9

[6] E. Anceaume, Y. Busnel, and N. Rivetti. 2015. Estimating the Frequency of Data Items in Massive Distributed Streams. In Proceedings of the 4th IEEE Symposium on Network Cloud Computing and Applications (NCCA). 59-66. https://doi.org/10.1109/NCCA. 2015.19

[7] E. Anceaume, Y. Busnel, N. Rivetti, and B. Sericola. 2015. Identifying Global Icebergs in Distributed Streams. In Proceedings of the 34th IEEE Symposium on Reliable Distributed Systems (SRDS). 266275. https://doi.org/10.1109/SRDS.2015.19 
[8] Kenneth R. W. Brewer. 1975. A simple procedure for

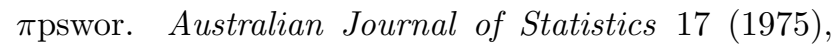
$166-172$.

[9] A. Chakrabarti, G. Cormode, and A. McGregor. 2007. A near-optimal algorithm for computing the entropy of a stream. In Proceedings of the 18th ACM-SIAM Symposium on Discrete Algorithms (SODA). 328-335.

[10] V. Chandola, A. Banerjee, and V. Kumar. 2009. Anomaly detection: A survey. Comput. Surveys 41, 3 (2009), 1-58.

[11] M. Charikar, K. Chen, and M. Farach-Colton. 2004. Finding frequent items in data streams. Elsevier Theoretical Computer Science 312, 1 (2004), 3-15.

[12] Guillaume Chauvet. 2012. On a characterization of ordered pivotal sampling. Bernoulli 18, 4 (2012), 13201340 .

[13] Guillaume Chauvet and Yves Tillé. 2006. A fast algorithm of balanced sampling. Journal of Computational Statistics 21 (2006), 9-31.

[14] G. Cormode and S. Muthukrishnan. 2005. An improved data stream summary: the count-min sketch and its applications. Journal of Algorithms 55, 1 (2005), 58-75.

[15] G. Cormode, S. Muthukrishnan, and K. Yi. 2008. Algorithms for distributed functional monitoring. In Proceedings of the 19th ACM-SIAM Symposium On Discrete Algorithms (SODA).

[16] M. Datar, A. Gionis, P. Indyk, and R. Motwani. 2002. Maintaining Stream Statistics over Sliding Windows. SIAM J. Comput. 31, 6 (2002), 1794-1813.

[17] Jean-Claude Deville. 1998. Une nouvelle (encore une!) méthode de tirage à probabilités inégales. Technical Report 9804. Méthodologie Statistique, Insee, Paris.

[18] Jean-Claude Deville and Yves Tillé. 1998. Unequal probability sampling without replacement through a splitting method. Biometrika 85 (1998), 89-101.

[19] Jean-Claude Deville and Yves Tillé. 2004. Efficient balanced sampling: The cube method. Biometrika 91 (2004), 893-912.

[20] Wayne Arthur Fuller. 1970. Sampling with random stratum boundaries. Journal of the Royal Statistical Society B32 (1970), 209-226.
[21] S. Ganguly, M. Garafalakis, R. Rastogi, and K. Sabnani. 2007. Streaming Algorithms for Robust, RealTime Detection of DDoS Attacks. In Proceedings of the 27th International Conference on Distributed Computing Systems (ICDCS 'OY). IEEE Computer Society, 4.

[22] Jaroslav Hájek. 1971. Discussion of an essay on the logical foundations of survey sampling, part on by D. Basu. In Foundations of Statistical Inference, Vidyadhar Prabhakar Godambe and D. A. Sprott (Eds.). Holt, Rinehart, Winston, Toronto, 326.

[23] D. M. Kane, J. Nelson, and D. P. Woodruff. 2010. An Optimal Algorithm for the Distinct Element Problem. In Proceedings of the 29th ACM Symposium on Principles of Database Systems (PODS).

[24] V. Karamcheti, D. Geiger, Z. Kedem, and S. Muthuskrishnan. 2005. Detecting malicious network traffic using inverse distribution of packet contents. In Proceedings of the 1st Workshop on Mining Network Data (MineNet).

[25] B. Krishnamurthy, S. Sen, Y. Zhang, and Y. Chen. 2003. Sketch-based Change Detection: Methods, Evaluation, and Applications. In Proceedings of the $3 \mathrm{rd}$ ACM SIGCOMM Internet Measurement Conference (IMC). 234-247.

[26] A. Lakhina, M. Crovella, and C.Diot. 2005. Mining Anomalies Using Traffic Feature Distributions. In Proceedings of the 28th ACM Conference on Applications, Technologies, Architectures, and Protocols for Computer Communications (SIGCOMM).

[27] Alina Matei and Yves Tillé. 2016. The R sampling package, Version 2.8. Université de Neuchâtel https://cran.r-project.org/ web/packages/sampling/index.html.

[28] A. Metwally, D. Agrawal, and A. El Abbadi. 2005. Efficient Computation of Frequent and Top-k Elements in Data Streams. In Proceedings of the 10th International Conference on Database Theory (ICDT).

[29] S. Muthukrishnan. 2005. Data Streams: Algorithms and Applications. Now Publishers Inc.

[30] O. Salem, S. Vaton, and A. Gravey. 2010. A scalable, efficient and informative approach for anomalybased intrusion detection systems: theory and practice. International Journal of Network Management 20, 5 (2010), 271-293.

[31] Yves Tillé. 2006. Sampling Algorithms. Springer, New York. 